\title{
Interdisziplinäre Zusammenarbeit für eine diskriminierungsfreie(re) Arbeitsbewertung*
}

\author{
Dr. jur. Regine Winter \\ Mitglied der Kommission Arbeits-, Gleichstellungs- und Wirt- \\ schaftsrecht des djb; Richterin am Bundesarbeitsgericht, Erfurt
}

\begin{abstract}
Nach wie vor ein Prinzip ohne Praxis: Entgeltgleichheit
Die verhältnismäßig schlechte Bezahlung in vielen „Frauenberufen“ wird im Vergleich zu „Männerberufen“ - beides als statistischer Befund und nicht als inhaltliche Zuschreibung gemeint - von Betroffenen nicht selten als „irgendwie“ ungerecht empfunden. Dies wird jedoch selten als Rechtsproblem, das mit rechtlichen Mitteln lösbar ist, aufgefasst.
\end{abstract}

„Gleiches Entgelt für gleichwertige Arbeit“ ohne Diskriminierung aufgrund des Geschlechts lautet der dafür einschlägige Rechtsanspruch im geschriebenen Recht ${ }^{1}$, der vor jedem Arbeitsgericht einklagbar ist. Er ist nicht neu, und trotzdem sind seine Tragweite und seine Wirkungsfähigkeit verhältnismäßig unbekannt. Dies trifft nicht nur für potenziell Betroffene zu. In der bei den Arbeitsgerichten ankommenden anwaltlichen Praxis spielt dieser Rechtsanspruch so gut wie gar keine Rolle, noch nicht einmal als Zweitbegründung in Eingruppierungsrechtsstreitigkeiten betreffend Frauen in sogenannten Frauenberufen. Dieser Rechtsanspruch ist in Deutschland im Wesentlichen ein „Prinzip ohne Praxis“. Die Gründe dafür sind vielfältig und vielschichtig.

Kein Grund liegt im mangelnden Bedarf. Entgeltsysteme einschließlich Tarifverträge sind in Deutschland nicht frei von struktureller Entgeltdiskriminierung. Zum Beleg dafür statt vieler ein Zitat aus dem „Dossier Entgeltungleichheit zwischen Frauen und Männern in Deutschland“ des Bundesministeriums für Familie, Senioren, Frauen und Jugend: „Dass Entgeltregelungen und ihre Anwendung Arbeit von Frauen unterbewerten können, belegen wissenschaftliche Untersuchungen, Praxiserfahrungen und nicht zuletzt Entscheidungen des Europäischen Gerichtshofes und deutscher Arbeitsgerichte“.2 Dagegen scheint das geltende Recht bisher machtlos zu sein.

Problematisch ist insbesondere die Rechtsdurchsetzung. Sie hängt von Klagen der Betroffenen ab. Einzelklagen sind im Bereich der Entgeltdiskriminierung bei gleichwertiger Arbeit besonders schwierig, denn es geht um tief in die Strukturen der Entgeltsysteme eingewobene Diskriminierung. Akteure der Entgeltpolitik, darunter die Tarifvertragsparteien, werden nach bisher geltender Rechtslage nicht wirkungsvoll zur Beendigung mittelbar diskriminierender Entgeltungleichbehandlung in die Pflicht genommen. ${ }^{3}$ Im Gegenteil ging in der Vergangenheit die Auslegung der grundgesetzlich garantierten Tarifautonomie in der BRD teilweise so weit, (mittelbar) diskriminierende Entgeltungleichbehandlung als von ihr gedeckt anzusehen oder jedenfalls zu meinen, einer aktiven Rechtspolitik zur Beendigung von Entgeltdiskriminierung stünde Artikel 9 Absatz 3 GG entgegen.
In diesem Beitrag geht es im Wesentlichen um eine weitere Ebene der vielfältigen Hindernisse der Rechtsverwirklichung: Wie kann verschiedenartige Arbeit im Hinblick auf eventuelle Gleichwertigkeit überhaupt verglichen werden? Heißt das nicht, „Äpfel mit Birnen“ zu vergleichen? Was soll der Maßstab des Vergleichs sein? Um einer Antwort auf diese Fragen näher zu kommen, erscheint mir der Blick über den Tellerrand der Rechtswissenschaft(en) hilfreich. Mein folgendes „Plädoyer" für eine inter-/transdisziplinäre Herangehensweise hat seinen „persönlichen“ Ursprung in einem jahrelangen Diskurs mit Kolleginnen aus der Wirtschaftswissenschaft (Personalpolitik/Management) und Sozialforschung, insbesondere mit Prof. Dr. Gertraude Krell ${ }^{4}$, der dieser Beitrag in erster Version als Festschriftaufsatz gewidmet war, und Dr. Karin Tondorf.

\section{„Gleichwertigkeit von Arbeit" - ein eher unverstandener Rechtsbegriff}

Der Anspruch auf gleiches Entgelt unabhängig vom Geschlecht für gleiche Arbeit ist seit Unterzeichnung der Römischen Verträge zur Gründung der EWG im Jahre 1957 vertraglich festgelegt (damals als Art. 119 EWG, später Art. 141 EG). In der Rechtsprechung des EuGH wurde dies früh auch als Anspruch auf gleiches Entgelt bei gleichwertiger Arbeit verstanden. Seit Jahrzehnten heißt es im Unionsrecht nunmehr

* Nachdruck (Festschrift Krell) mit kleinen Änderungen mit freund-
licher Genehmigung des Rainer Hampp Verlags, http://www.
Hampp-Verlag.de.
1 Näher Winter, Regine, „\$ 1 TVG“ (Teilkommentierung, darin: Verbot
der Entgeltdiskriminierung, Rn. 489 ff., 510), in: Däubler (Hg.): Tarif-
vertragsgesetz mit Arbeitnehmer-Entsendegesetz. Kommentar, 3.
Aufl., Baden-Baden 2012; dies., Gleiches Entgelt für gleichwertige
Arbeit - Ein Prinzip ohne Praxis, Baden-Baden 1998; Feldhoff, Ker-
stin, Der Anspruch auf gleichen Lohn für gleichwertige Arbeit, Ba-
den-Baden 1998.

2 Bundesministerium für Familie, Senioren, Frauen und Jugend (BMFSFJ), Dossier Entgeltungleichheit zwischen Frauen und Männern in Deutschland, Osnabrück 2009, S. 21.

3 Vgl. näher Pfarr, Heide, „Entgeltgleichheit in kollektiven Entgeltsystemen - Aufgabe für die Tarifparteien, die Rechtsprechung, aber auch die Gesetzgebung“, in: Oetker, Hartmut/Preis, Ulrich/Rieble, Volker (Hg.): 50 Jahre Bundesarbeitsgericht, Festschrift, München 2004, S. 779-794; dies., ,Die Entgeltgleichheit für Frauen und Männer erfordert ein Durchsetzungsgesetz “, WSI-Mitteilungen 2011, 64 (5), S. 253256; Winter, Regine, a.a.O. (1998, Fn. 1); dies.,

„Diskriminierungsfreie(re) Entgeltgestaltung - leider immer noch ein Prinzip ohne Praxis“, in: Hohmann-Dennhardt, Christine/Körner, Marita/Zimmer, Reingard (Hg.): Geschlechtergerechtigkeit. Festschrift für Heide Pfarr, Baden-Baden 2010, S. 320-333; vgl. auch Jochmann-Döll, Andrea/Tondorf, Karin, Diskriminierungsfreie Tarifverträge, edition der Hans-Böckler-Stiftung, Nr. 151, Düsseldorf 2009.

4 Ausdruck dieses Austausches ist z.B. Krell, Gertraude/Winter, Regine, „Anforderungsabhängige Entgeltdifferenzierung. Orientierungshilfen auf dem Weg zu einer diskriminierungsfreieren Arbeitsbewertung“, in: Krell, Gertraude/Ortlieb, Renate/Sieben, Barbara (Hg.): Chancengleichheit durch Personalpolitik. Gleichstellungen von Frauen und Männern in Unternehmen und Verwaltungen. Rechtliche Regelungen - Problemanalysen - Lösungen, 6. Aufl., Wiesbaden 2011, S. 343-360. 
ausdrücklich, dass gleiches Entgelt für Männer und Frauen bei gleicher oder gleichwertiger Arbeit sicherzustellen ist (mittlerweile in Art. 157 des Vertrages über die Arbeitsweise der Europäischen Union - AEUV).

Immerhin 26 Jahre lang, nämlich von August 1980 bis zum Inkrafttreten des AGG im August 2006, stand „gleiches Entgelt für gleichwertige Arbeit“ unabhängig vom Geschlecht als Rechtsanspruch auch im BGB, nämlich in $\mathbb{S} 612$ Absatz 3. Dorthin war er nicht auf Initiative des deutschen Gesetzgebers, sondern auf europäischen Druck in der Verpflichtung zur Umsetzung von Recht der damaligen Europäischen Wirtschaftsgemeinschaft (EWG) gekommen. Es kann sein, dass bei dieser Umsetzung in nationales Recht, die verspätet und widerstandsbegleitet erfolgte, das Verständnis des Begriffes der gleichwertigen Arbeit auf dem Weg von der europäischen auf die nationale Ebene steckengeblieben ist.

Auch wenn dies berücksichtigt wird, ist die weitere Entwicklung - vielmehr „Nicht-Entwicklung“ - einigermaßen erstaunlich. Beispielsweise Ende der 1980er/Anfang der 1990er Jahre, als der Entgeltgleichheitsanspruch im BGB immerhin schon ca. zehn Jahre geltendes Recht war und im (damaligen) EWG/EG-Recht immerhin schon mehr als dreißig Jahre zählte, war er bei uns nahezu unbekannt. Mit Ausnahme einzelner Stimmen in der juristischen Literatur ${ }^{5}$ war im juristischen Mainstream der Gesetzeskommentierung lange kaum Hilfreiches zum Begriff der gleichwertigen Arbeit lesbar. Dies verwundert - ist doch juristische Gesetzeskommentierung bekanntlich geübt darin, Begriffen und Begriffsunterschieden präzise auf den Grund zu gehen. Nicht aber hier. Beispielsweise erwähnten nur wenige Stimmen der Kommentarliteratur, dass $\ 612$ Absatz 3 BGB zwei Vergleichsfacetten enthielt, nämlich „gleiche“ „oder“ „gleichwertige“ Arbeit, und dass auch ein Vergleich verschiedenartiger Arbeit im Hinblick auf eventuell gleichen Wert gemeint war. Soweit Letzteres überhaupt Erwähnung fand, wurde solch ein Vergleich eher für nicht handhabbar gehalten. So war beispielsweise 1992 im Münchener Handbuch zum Arbeitsrecht zu lesen, regelmäßig fehle die Vergleichsmöglichkeit, wenn bestimmte Tätigkeiten nur von Frauen oder nur von Männern ausgeübt würden. ${ }^{6} \mathrm{Als}$ es im Jahre 1993 in einem Urteil des EuGH zu Artikel 119 EWG um die Frage der Entgeltdiskriminierung und den Vergleich von zwei unterschiedlichen Tätigkeiten im britischen National Health Service (Logopädin und leitender Pharmazeut) ging ${ }^{7}$, war anschließend in der deutschen juristischen Literatur große Verwunderung erkennbar, dass ein rechtsförmiger Vergleich von verschiedenartigen Tätigkeiten auf ihren Wert hin überhaupt möglich sein kann. ${ }^{8}$

Tiefergehende Orientierung zum Begriff der gleichwertigen Arbeit boten damals in der BRD insbesondere Stimmen außerhalb des juristischen Diskurses, insbesondere Gertraude Krell ${ }^{9}$, Barbara Dürk ${ }^{10}$, Andrea Jochmann-Döll11 und Kooperationen dieser Autor/inn/en ${ }^{12}$.

Ein Grund für die „Nicht-Entwicklung“ des eigentlich deutlich formulierten Rechtsanspruchs auf gleiches Entgelt bei gleichwertiger Arbeit mag damit zusammenhängen, dass das
Konzept eine inter- oder auch transdisziplinäre Herangehensweise erfordert und allein juristisches Handwerkszeug weder für sein Verständnis noch für seine Bearbeitung ausreicht. Dass es der Rechtsanspruch auf „gleiches Entgelt für gleichwertige Arbeit“ erlaubt, nicht nur sehr ähnliche Arbeit zu vergleichen, sondern dass ein Vergleich verschiedenartiger Arbeit im Hinblick auf den Wert möglich und geboten ist, erschließt sich nur, wenn für diese Bewertung ein gemeinsamer Maßstab ersichtlich ist und herangezogen wird. Ohne gemeinsamen Maßstab ist ein rationaler Vergleich nicht möglich. Im rein juristischen Repertoire ist ein dafür geeigneter Maßstab aus sich heraus nicht verfügbar. Vorfindbar ist er jedoch im ersten Zugriff beispielsweise in tarifvertraglichen Eingruppierungskatalogen und anderen Entgeltsystemen, wo verschiedenartige Arbeit im Ergebnis auf ihre Gleichwertigkeit miteinander verglichen ist, beispielsweise durch gemeinsame Platzierung in ein und derselben Entgeltgruppe. Sind dafür abstrakte Oberbegriffe genannt, sind sie erster Anhaltspunkt für darüber hinausgehende rechtsförmige Gleichwertigkeitsvergleiche. ${ }^{13}$ Enthält ein Entgeltsystem keine Oberbegriffe oder sind die vorhandenen selbst nicht frei von Diskriminierung, dann kann eine Erweiterung des Blickes auf arbeitsbewertendes Know-how der Personalwirtschaft hilfreich sein. Rechtsförmige Gleichwertigkeitsvergleiche von Arbeit als operationalisierbares und lösbares Problem zu begreifen, erfordert ein arbeitsbewertendes Grundverständnis mit offenem Blick für Diskriminierungspotentiale.

5 Z.B. Colneric, Ninon, Leichtlohngruppen und Gleichbehandlungsgebot, Arbeitsrecht im Betrieb, 1986, 7 (2), S. 40-42; dies., Gleichberechtigung von Mann und Frau im Europäischen Gemeinschaftsrecht, Betriebs-Berater 1988, 46 (14), S. 968-976; Pfarr, Heide/Bertelsmann, Klaus, Diskriminierung im Erwerbsleben - Ungleichbehandlungen von Frauen und Männern in der Bundesrepublik Deutschland, Baden-Baden 1989.

6 Richardi, Reinhard, „Gleichberechtigung von Mann und Frau“, in: Richardi, Reinhard/Wlotzke, Otfried (Hg): Münchener Handbuch zum Arbeitsrecht, Band 1, Individualarbeitsrecht I, § 11, München 1992, S. 102-116, Rn. 40.

7 Urteil „Enderby“ vom 27. Oktober 1993-C-127/92 - NZA 1994, 797.

8 Nachweise bei Winter, Regine, a.a.O. (1998, Fn. 1), S. $21 \mathrm{ff}$.

9 Z.B. Krell, Gertraude, Das Bild der Frau in der Arbeitswissenschaft, Frankfurt a.M./New York 1984; dies., „Entgeltdiskriminierung durch Arbeitsbewertung und Eingruppierung“, 1990; dies., „Die Verfahren der Arbeitsbewertung - Kritische Bestandsaufnahme und Perspektiven“, in: Winter, Regine (Hg.): Frauen verdienen mehr. Zur Neubewertung von Frauenarbeit im Tarifsystem, Berlin 1994, S. 43-55.

10 Z.B. Dürk, Barbara, Wenn das Brunnenmädchen im Heilbad den Hahn zudreht, WSI-Mitteilungen 1991, 44 (12), S. 724-733.

11 Jochmann-Döll, Andrea, Gleicher Lohn für gleichwertige Arbeit: Ausländische und deutsche Konzepte und Erfahrungen, München/ Mering 1990.

12 Z.B. Jochmann-Döll, Andrea/Krell, Gertraude, Die Methoden haben gewechselt, die „Geschlechtsabzüge“ bleiben - Auf dem Weg zu einer Neubewertung von Frauenarbeit?, in: Hausen, Karin/Krell, Gertraude (Hg.): Frauenerwerbsarbeit. Forschungen zu Geschichte und Gegenwart, München/Mering 1993, S. 133-148; dies., „Leistungsabhängige Entgeltdifferenzierung auf dem gleichstellungspolitischen Prüfstand“, in: Krell, Gertraude/Ortlieb, Renate/ Sieben, Barbara (Hg.): Chancengleichheit durch Personalpolitik, 6. Aufl., Wiesbaden 2011.

13 Für ein praktisches Beispiel vgl. Winter, Regine, „Ausbildung, Arbeitsanforderungen und Tarifvertrag: Erzieherinnen tragen (k)eine Verantwortung?“, in: Winter, Regine (Hg.): Frauen verdienen mehr. Zur Neubewertung von Frauenarbeit im Tarifsystem, Berlin 1994, S. $247-268$ (259 ff.). 
Seit Inkrafttreten des AGG ist das juristische Verstehen zum Thema der Entgeltgleichheit bei gleichwertiger Arbeit nicht beträchtlich größer geworden. Das AGG hat für diesen Rechtsanspruch, der unionsrechtlich nach wie vor unverändert besteht und damit auch auf nationaler Ebene rechtlich durchgesetzt werden kann, eher eine Verschlechterung gebracht: Im AGG ist dieser Rechtsanspruch nicht ausdrücklich formuliert und damit relativ „unsichtbar“. Der frühere $\$ 612$ Absatz 3 BGB ist seitdem nicht mehr in Kraft. Damit ist die ehemals klare Aussage „darf für gleiche oder gleichwertige Arbeit nicht wegen des Geschlechts des Arbeitnehmers eine geringere Vergütung vereinbart werden als bei einem Arbeitnehmer des anderen Geschlechts“ aus dem BGB verschwunden, jedoch im AGG nicht vergleichbar ausdrücklich formuliert worden. Es verwundert kaum, dass dementsprechend der kommentierende Widerhall dieses Rechtsanspruchs in den einschlägigen Kommentaren zum AGG relativ dürftig ist. Dies beginnt schon damit, dass dieses Thema, da im AGG kein Paragraph ausdrücklich davon handelt, auch in den Kommentierungen keinen „Ort“ hat, an dem es einheitlich zu finden wäre.

\section{Quo vadis?}

Der Stand der Dinge ist heute - mehr als fünfzig Jahre nach Unterzeichnung der Römischen Verträge - in Deutschland also bisher keine Erfolgsgeschichte. Gibt es derzeit Grund zu der Annahme, dass sich daran etwas ändert? Jedenfalls ist einiges an Bewegung und Diskussion bis hin zu Gesetzgebungsinitiativen zu verzeichnen, damit aus dem Rechtsanspruch auf Entgeltgleichheit Rechtswirklichkeit wird. ${ }^{14}$

Arbeit nicht abhängig vom Geschlecht oder einem anderen verbotenen Differenzierungsgrund wie Alter, Behinderung oder Herkunft zu bewerten, ist ein Gebot der Fairness. All diese Gebote der Fairness sind als Diskriminierungsverbote geltendes Recht. Ein Mehr an inter-/transdisziplinärer Zusammenarbeit - angefangen beispielsweise bei Schulungsveranstaltungen - kann aus meiner Sicht einen Beitrag dazu leisten, daraus Rechtswirklichkeit werden zu lassen.

14 Vgl. dazu insbesondere Pfarr (2011, Fn. 3) sowie Pfarr und weitere Beiträge in dieser djbz.

\section{Aktuelle Entwicklungen auf EU-Ebene zur Bekämpfung von Entgeltdiskriminierung}

\section{(Auswahl - Stand Juni 2012)}

Entschließung des Europäischen Parlaments vom 24. Mai 2012 mit Empfehlungen an die Kommission zur Anwendung des Grundsatzes des gleichen Entgelts für Männer und Frauen bei gleicher oder gleichwertiger Arbeit (2011/2285(INI)).

- Daraus (Auszug unter Auslassung einzelner Punkte):

Das Europäische Parlament

- fordert die Kommission auf, bis spätestens 15. Februar 2013 die Richtlinie 2006/54/EG zu überprüfen und Änderungen im Sinne von Artikel 157 AEUV unter Beachtung der dieser Entschließung beigefügten ausführlichen Empfehlungen zumindest in Bezug auf die folgenden Aspekte des geschlechtsbezogenen Lohngefälles vorzuschlagen:

- Begriffsbestimmungen,

- Analyse der Situation und Transparenz der Ergebnisse,

- Arbeitsplatzbewertung und berufliche Einstufung,

- Gleichstellungsgremien und Rechtsmittel,

- sozialer Dialog,

- Verhütung von Diskriminierung,

- Gender Mainstreaming,

- Sanktionen,

- Straffung der Unionsregelungen und -politik.

- ist sich darüber im Klaren, dass es vielfältige Gründe für eine Verschärfung des Lohngefälles gibt, und stellt deshalb fest, dass ein Ansatz, der mehrere Ebenen und mehrere Facetten einbezieht, der Union eine starke Führungsrolle bei der Koordination der Maßnahmen, der Förderung bewährter Methoden und dem Einbinden verschiedener Handlungsträger, zum Beispiel der europäischen Sozialpartner und nichtstaatlicher Organisationen, abverlangt und das Ziel verfolgt, eine euro- paweite Strategie zur Bekämpfung des geschlechtsspezifischen Lohngefälles zu entwickeln;

- fordert die Mitgliedstaaten auf, die neu gefasste Richtlinie 2006/54/EG einheitlich umzusetzen und durchzusetzen, den Privatsektor wie auch den öffentlichen Sektor anzuregen, eine aktivere Rolle beim Abbau des geschlechtsspezifischen Lohngefälles zu spielen; ist der Ansicht, dass die Mitgliedstaaten und die Kommission die Sozialpartner einschließlich der Arbeitgeber auffordern sollten, Arbeitsbewertungssysteme zu schaffen, die geschlechtsneutral sind, Systeme der beruflichen Einstufung umzusetzen und das Konzept des Arbeitsplatzes mit gleichem Entgelt zu unterstützen;

- fordert die Mitgliedstaaten auf, bei der Bekämpfung der Unterschiede im Bereich des Entgelts, von denen Frauen in den öffentlichen Verwaltungen, Einrichtungen und Unternehmen generell betroffen sind, mit gutem Beispiel voranzugehen;

- betont, wie wichtig Tarifverhandlungen und Tarifverträge beim Kampf gegen die Diskriminierung von Frauen sind, insbesondere in den Bereichen Zugang zur Beschäftigung, Löhne, Arbeitsbedingungen, beruflicher Aufstieg und Berufsbildung;

- begrüßt die Initiative der Kommission zur Einführung eines Europäischen Tags der Lohngleichheit, der erstmals am 5. März 2011 stattfand und zum zweiten Mal am 2. März 2012 begangen wurde;

- stellt fest, dass Lohnunterschiede, die auf andere Faktoren wie Rasse, ethnische Zugehörigkeit, sexuelle Ausrichtung oder Religion zurückzuführen sind, nicht hingenommen werden dürfen; 